\title{
Effect of recombinant bovine basic fibroblast growth factor gel on repair of rosacea skin lesions: A randomized, single-blind and vehicle-controlled study
}

\author{
YANG LUO, XIU-LI LUAN, YU-JIAO SUN, LI ZHANG and JIAN-HONG ZHANG \\ Department of Dermatology, Lanzhou General Hospital, Lanzhou, Gansu 730050, P.R. China
}

Received March 3, 2018; Accepted January 31, 2019

DOI: $10.3892 /$ etm.2019.7258

\begin{abstract}
The aim of the present study was to assess the effect of topical use of recombinant bovine basic fibroblast growth factor (rbFGF) gel on the repair of facial skin lesions in patients with rosacea. In the present single-blind study, a total of 1,287 patients with Demodex mite-induced rosacea who received treatment with ornidazole tablets were randomized to rbFGF gel treatment group $(n=651)$ or control group $(n=636)$ without revealing the group identity. Patients in the treatment group were treated with topical application of rbFGF gel over the skin lesions $\left(0.2 \mathrm{~g} / \mathrm{cm}^{2}\right)$ for up to 8 weeks, whereas patients in the control group received gel vehicle treatment unless ulceration occurred. Skin lesions of all patients were scored prior to and following treatment with rbFGF gel and subjected to histological analysis. All patients were followed up for 6 months. Significant improvement in the total effective rates for erythema, papules, desquamation and dryness were observed in the rbFGF treatment group. At the end of the 2, 4 and 6 months of follow-up, the total effective rates for patients in the treatment group were significantly higher than those in the control group ( 81.67 vs. $28.84 \%$; 85.11 vs. $40.81 \%$, and 96.56 vs. $55.82 \%$, respectively). Following treatment for 6 months, none of the patients in the rbFGF group exhibited ulceration or scar formation. In the control group, $61 \%$ of patients experienced exacerbation of skin lesions, of which, $12 \%$ exhibited ulceration and were treated with rbFGF gel to prevent scar formation. Histological analysis revealed gradual reduction in epidermal hyperplasia and resolution of dermal edema in skin lesions treated with rbFGF gel. In conclusion, rbFGF gel may improve the repair of facial rosacea skin lesions in patients treated with anti-Demodex.
\end{abstract}

Correspondence to: Dr Yang Luo, Department of Dermatology, Lanzhou General Hospital, 333 Binhenan Road, Lanzhou, Gansu 730050, P.R. China

E-mail: lytmmu@163.com

Key words: recombinant bovine basic fibroblast growth factor, rosacea, Demodex, dermis, restore

\section{Introduction}

Rosacea is a common chronic inflammatory skin disease, which affects facial skin blood vessels and sebaceous glands. The primary symptoms include transient or non-transient erythema, papules, pustules and telangiectasia, all of which largely affect the central face. Patients with a prolonged disease course may develop dark red plaques or rhinophyma (typical irregular thickening of the nasal skin) due to hyperplasia (1). According to the National Rosacea Society Expert Committee, rosacea is classified as mild, moderate or severe based on severity of the lesions and the symptoms (2). The protracted disease course, tendency for recurrence, associated changes in facial complexion, skin ulceration and scar formation tend to have a detrimental effect on the physical, mental and social health of the patients (3).

Primary treatment modalities for rosacea at present include anti-inflammatory, antipruritic and anti-parasitic agents, and vasomotor regulation (2). Current treatments for rosacea mainly focus on alleviating the syndrome to improve the quality of life in patients, and preventing disease progression. However, they are not completely effective possibly due to a lack of clear understanding of the pathogenetic mechanisms of rosacea. More improved and novel alternative treatment options are in urgent need (4).

A close association of Demodex mite with facial dermatitis was previously observed (5). Demodex mite is a microscopic ectoparasite with a slender body (length, 0.3-0.4 mm), which mainly feeds off epidermal cells and sebum components. It inhabits human and mammalian hair follicles and sebaceous glands, especially the skin over the nose, cheeks, forehead and chin. The human body has characteristics of high infectivity, and low and conditional pathogenicity to Demodex mites $(6,7)$. The infestation rate of Demodex mite is also high in rosacea patients (5). If ornidazole treatment is used alone, untreated lesions tend to aggravate and result in ulceration and scar formation (Fig. 1) (8).

Fibroblast growth factor refers to a class of secreted polypeptide ligands that are widely used for treatment of burns (including superficial II, deep II, granulation wounds), chronic wounds (including chronic ulcer), fresh wounds (traumatic, donor site wound and surgical wounds), and for treatment of neurological diseases such as Parkinson's disease, and repair of corneal lesions (9-11). In our previous study, a statistically 

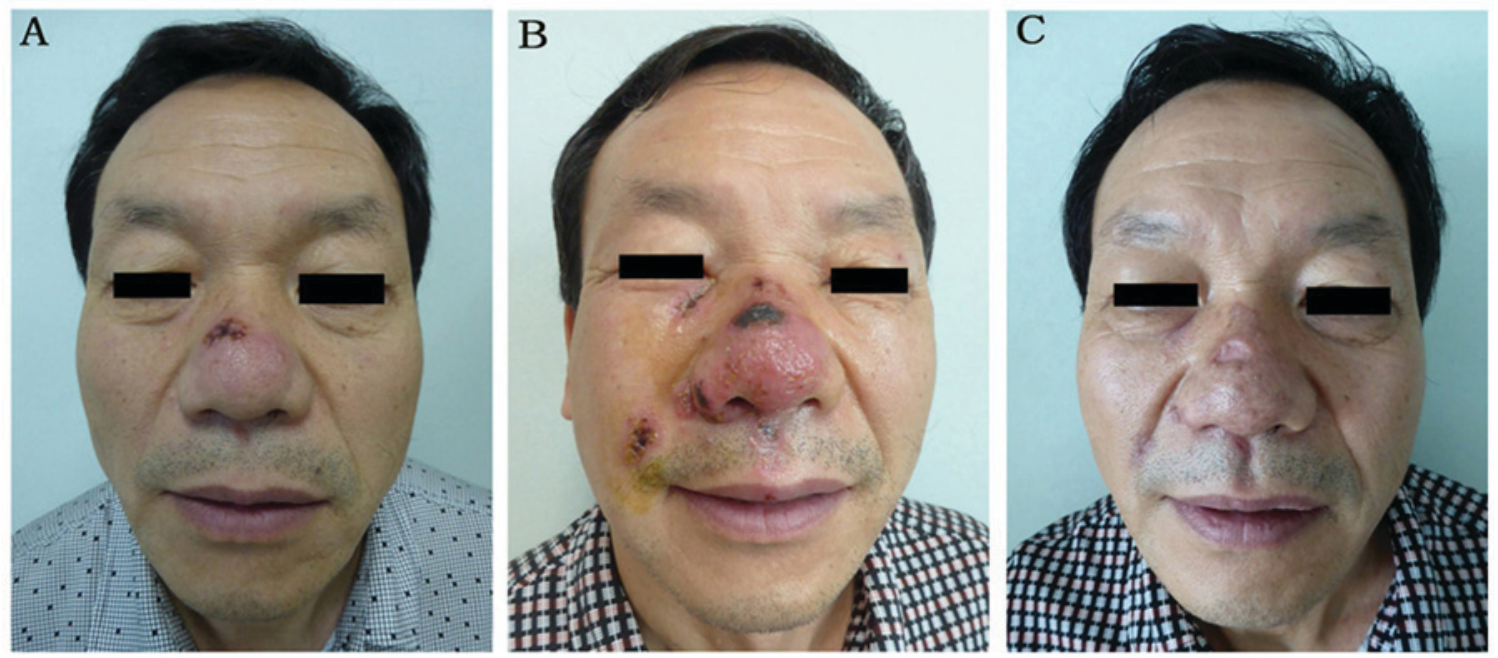

Figure 1. A male patient with rosacea who did not receive rbFGF treatment developed ulceration and scar formation in the lesion. This patient was not included in the present study but this case inspired the use of rbFGF to treat the complications, including ulceration and scar formation, due to rosacea. (A) When admitted, the patient had facial lesions with erythema and papules. (B) Following treatment with ornidazole for 1 week, the lesion was aggravated with increased lesion area and necrosis. (C) Following 8 weeks of ornidazole treatment, erythema and papules had faded and a scar remained. rbFGF, recombinant bovine basic fibroblast growth factor.

significant beneficial effect of topical use of recombinant bovine basic fibroblast growth factor (rbFGF) gel was observed in patients with facial dermatitis (8). However, whether rbFGF improves treatment outcomes in rosacea patients following anti-Demodex treatment remains unknown. In the present study, the treatment outcomes of rbFGF in 1,287 rosacea patients following anti-Demodex treatment were investigated and significant improvement was observed in the total effective rates for erythema, papules, desquamation and dryness in the rbFGF treatment group.

\section{Materials and methods}

Subjects. The present study was approved by the Ethics Committee of Lanzhou General Hospital (Lanzhou, China). In the present single-blind randomized study, a total of 1,287 patients with Demodex-mite induced rosacea were treated at the dermatology outpatient department of Lanzhou General Hospital between June 2014 and February 2016. The study was focused on Demodex-mite induced rosacea manifested with papules and pustule. The inclusion criteria were clinical manifestations of rosacea, which is defined as persistent erythema of the central portion of the face, lasting for at least 3 months (detailed diagnostic criteria for rosacea are listed in Table I) (2) and positive test results for Demodex mite from a standardized skin surface biopsy. All patients recruited in the present study were classified as moderate to severe rosacea (12). As all patients exhibited moderate to severe rosacea with primary features including transient and non-transient erythema, papules and pustules and telangiectasia, the patients were not divided into subtypes. The exclusion criteria were pregnant or lactating women; patients who had cardiovascular, liver, kidney or hematopoietic system disorders and/or other serious primary diseases, such as heart disease and diabetes; patients with mental illness or cancer; patients who had been diagnosed and previously treated for rosacea; and patients who were allergic to ornidazole or rbFGF gel.
Table I. Diagnostic criteria for rosacea, including primary and secondary features.

features (presence of one or more of the following)

Secondary features (may include one or more of the following)

Flushing (transient erythema) Burning or stinging discomfort Nontransient erythema Papules and pustules Telangiectasia Dry appearance and texture Edema Ocular manifestations Peripheral location Phymatous changes Plaque

Informed consent was obtained from all enrolled patients. The clinical trial registration number was ChiCTR-IPR-15006451.

Standardized skin surface biopsy. Patients cleaned their faces with gentle cleanser and water prior to the biopsy. When the skin dried, a sterile sharp blade was scrapped on adjacent areas on the patients' nose. The accumulated debris or sebaceous secretions were collected on a glass slide, a drop of 5\% potassium hydroxide was added and the coverslip was applied. The samples were then o observed under a light microscope (magnification, $\mathrm{x} 40$ ).

Bacterial culture. The accumulated debris was plated on an agar plate (Autobio Diagnostics Co., Ltd., Zhengzhou, China) and incubated at $35^{\circ} \mathrm{C}$ for $18-24 \mathrm{~h}$. Staphylococci colonies are usually middle-sized, spherical, flat and smooth, moist with smooth edges, they are golden-yellow, white, milky white or lemon-yellow, and some colonies possess $\beta$-hemolytic rings. Based on the aforementioned characteristics, the authors further separated the colonies that were considered to be staphylococci 


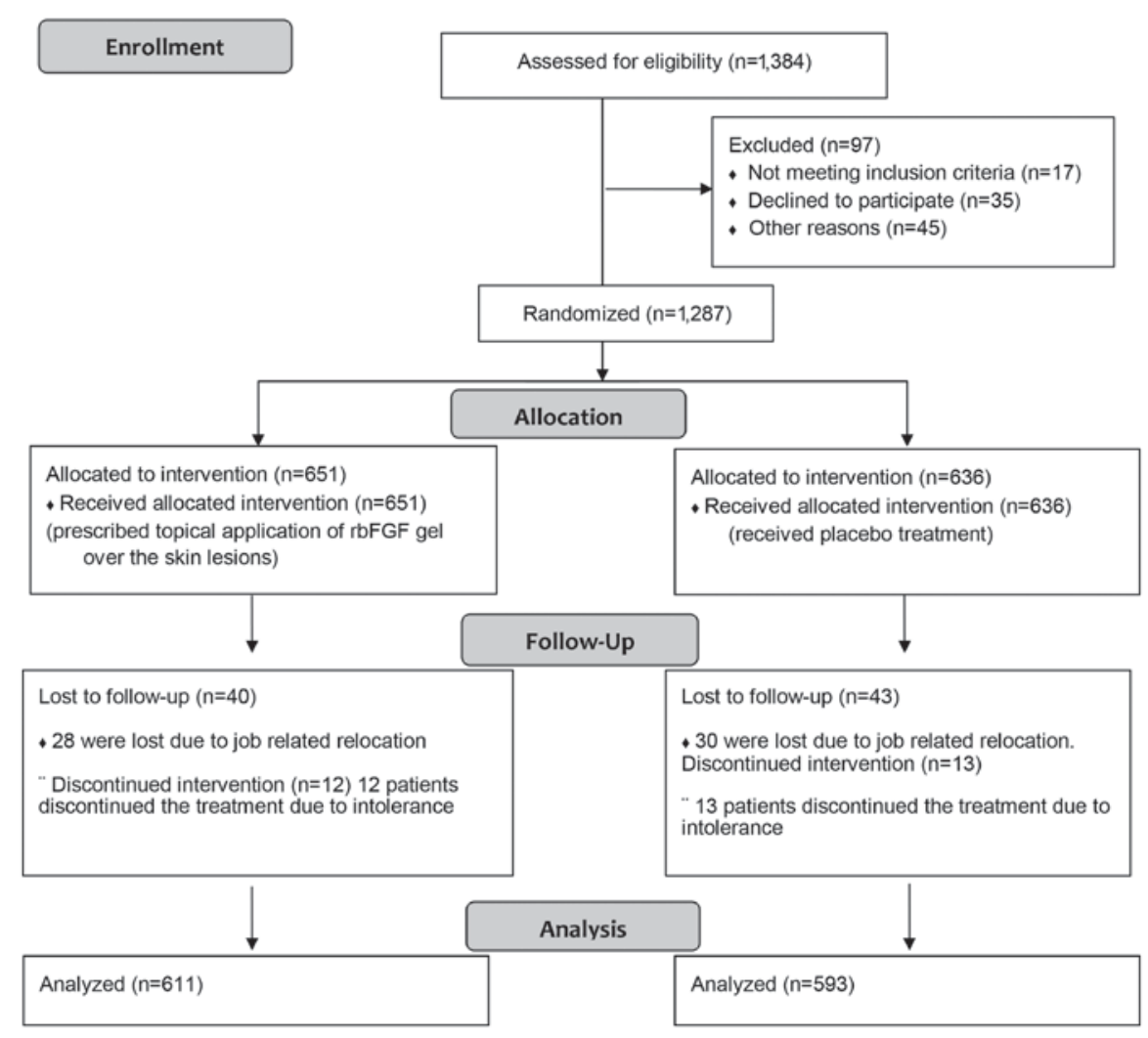

Figure 2. Schematic diagram of the experimental design.

and performed catalase tests after $48 \mathrm{~h}$ of culturing to confirm if they were. No staphylococci infections were identified.

Due to potential bacterial infection, 6 patients were treated with $2 \%$ mupirocin $\left(0.2 \mathrm{~g} / \mathrm{cm}^{2}\right.$, t.i.d; Sino-American Tianjin SmithKline \& French Laboratories, Ltd., Tianjin, China) for 5-10 days and 9 patients were treated with $2 \%$ fusidic acid ointment $\left(0.2 \mathrm{~g} / \mathrm{cm}^{2}\right.$, b.i.d; Bright Future Pharmaceutical Laboratories, Ltd., Hong Kong, China) for 1 to 2 weeks. If the experiment failed, patients were treatment with compound betamethasone injections $(5 \mathrm{mg} / \mathrm{ml}$ betamethasone dipropionate and $2 \mathrm{mg} / \mathrm{ml}$ betamethasone sodium phosphate; both Merck KGaA, Darmstradt, Germany) and rbFGF gel.

Treatment. The enrolled patients were prescribed oral ornidazole tablets ( $0.25 \mathrm{~g} /$ tablet; $500 \mathrm{mg}$ twice/day; Zhejiang Aisheng Pharmaceutical Co., Ltd., Zhejiang, China) for 2-4 courses (14 days/course). All patients were then randomly assigned to the treatment $(n=651)$ or control $(n=636)$ group (Fig. 2). Following treatment with ornidazole for 7 days, patients in the treatment group were prescribed topical application of rbFGF gel (21,000 IU/5 g; Zhuhai Yisheng Biological Pharmaceutical Co., Ltd., Zhuhai, China) over the skin lesions $\left(0.2 \mathrm{~g} / \mathrm{cm}^{2}\right)$ for 2-8 weeks, whereas patients in the control group received rbFGF gel vehicle treatment (containing heparin sodium, Carbopol ${ }^{\circledR} 934 \mathrm{P}$, and Triethanolamine which are the base for the rbFGF gel), unless ulceration occurred.

Total efficacy rate. All patients were scored by the same doctor following the ornidazole treatment for their primary features, including erythema, papules, desquamation, dryness and telangiectasia. Each of the features were counted separately. The results were graded as absent ( 0 score), mild (1 score), moderate (2 score) or severe (3 score) (12-14). Total efficacy rate and effective sample size were calculated using the following equation: Effective index $=$ [total score (before rbFGF treatment-after rbFGF treatment)/total score before treatment] x 100. The total efficacy index was graded according to the classification system shown in Table II.

Skin biopsy and histological analysis. As described previously (15), skin biopsy was taken in the clinic following rbFGF gel treatment. Biopsy tissues were fixed in $10 \%$ formalin (Shanghai Baoman Biotechnology Co., Ltd., Shanghai, China) for $12 \mathrm{~h}$ at room temperature, then dehydrated and embedded in paraffin (Shanghai Hualing Equipment Factory, Shanghai, China) and sectioned ( $4 \mu \mathrm{m})$ using a Leica section station (RM2016; Leica Microsystems GmbH, Wetzlar, Germany). The sections were then bleached using a pathological bleaching and drying machine (Changzhou Electronic Instrument Co., Ltd., Changzhou, China), dewaxed and stained with hematoxylin (Shanghai Chemical Reagent Co., Ltd., Shanghai, China) and eosin (HE; Third Factory of Shanghai Reagent Chemicals, Shanghai, China) for $15 \mathrm{~min}$ at room temperature. The HE-stained sections were observed with an optical microscope (BX53; Olympus Corporation, Tokyo, Japan) under a magnfication of $\times 40$ or $\times 100$ and diagnosed by a pathologist.

Follow up. Patients in both groups were followed up for 6 months. A total of 83 patients $(6.45 \%)$ were lost to follow-up and were excluded from the analysis. Among these, 58 were lost due to job-related relocation $(n=28$ from the rbFGF treatment group and $n=30$ from control group) and 25 patients 
Table II. Evaluation of clinical efficacy.

\begin{tabular}{lll}
\hline Grade & Effective index & \multicolumn{1}{c}{ Clinical manifestations } \\
\hline Effective & $\geq 90 \%$ & $\begin{array}{l}\text { A decrease in the majority of skin lesions, e.g., papules } \\
\text { and pus blister are not observed, nasal skin becomes } \\
\text { smooth and flat without protrusion, and pores } \\
\text { become small } \\
\text { Markedly improved }\end{array}$ \\
& $\geq 60 \%,<90 \%$ & $\begin{array}{l}\text { Majority of skin lesions subsided, e.g., papules, pus } \\
\text { basically disappeared, nasal skin has significantly } \\
\text { improved }\end{array}$ \\
Improved & & $\begin{array}{l}\text { Skin lesions have been reduced, e.g., more than half } \\
\text { of the papules, pus blisters subsided and nasal skin } \\
\text { lesions improved } \\
\text { Neffective }\end{array}$ \\
& $<20 \%,<60 \%$ & $\begin{array}{l}\text { No marked improvement in skin lesions, e.g., more } \\
\text { than half of the papules, pus blister remained and } \\
\text { nasal skin lesions remain unchanged }\end{array}$ \\
\hline
\end{tabular}

Scoring based on the manifestation: Absent (score, 0), mild (score, 1), moderate (score, 2) or severe (score, 3 ). Effective index $=$ [total score (prior to rbFGF treatment-following rbFGF treatment)/total score prior to treatment] x100. Effective sample size $=$ effective + markedly improved + improved. Total effective rate $=$ effective sample size/total sample size $\mathrm{x} 100$.

discontinued the treatment due to intolerance $(n=12$ from rbFGF treatment group and $n=13$ from control group).

A total of 71 patients in the control group were treated with rbFGF due to the occurrence of ulceration during follow-up. The lesions were scored at the end of the 2nd, 4 th and 6 th month, and the total effective rate of each lesion index was statistically analyzed. At the 2nd and 6th week of the rbFGF gel treatment, routine pathological examination of the facial lesions was performed to evaluate the rosacea skin lesions (15).

Statistical analysis. All data were analyzed using IBM SPSS 19.0 statistics software (IBM Corp., Armonk, NY, USA). Wilcoxon sign rank test was used to evaluate the lesion score prior to and following the rbFGF gel treatment. $\chi^{2}$ test was used to assess differences between groups. $\mathrm{P}<0.05$ was considered to indicate a statistically significant difference.

\section{Results}

A total of 1,204 [966 female (80.23\%) and 238 male (19.77\%)] with Demodex mite-induced rosacea were enrolled in the present study. The mean age of patients was 32.1 years (range, 18-60 years); the mean duration of disease course was 3.5 years (range, 0.1-20.0 years). Following treatment with ornidazole for 7 days, all patients were randomized to the rbFGF ( $n=611$; 496 female and 115 male; mean age, 31.8 years) or control $(n=593 ; 470$ female and 123 male; mean age, 32.0 years) groups. Retrospective images of the treatment and control groups are presented in Figs. 3 and 4, respectively. There were no marked differences with respect to baseline patient characteristics between the two groups (Figs. 3A and 4A). The pathological symptoms of rosacea included superficial dermal tissue necrosis, caseous necrosis and formation of granuloma structures. The current study demonstrated that the application of rbFGF gel can effectively repair the pathological structure of rosacea tissue.
In the pathological analysis of the tissue samples, neutrophil activity was not observed. Furthermore, there was no purulent discharge from the facial lesions. Culture of necrotic tissue secretions did not grow Staphylococcus aureus and Staphylococcus epidermidis (16). Finally, 6 patients were treated with $2 \%$ mupirocin for 5-10 days and 9 patients were treated with $2 \%$ fusidic acid ointment for 1 to 2 weeks; these treatments failed. Only treatments with compound betamethasone injections and rbFGF gel were effective.

All patients were followed up for at least 6 months. At 2-month follow up, $43.37 \%$ patients in the treatment group exhibited a marked improvement (Fig. 3B), compared with $23.95 \%$ patients in the control group (Fig. 4B). Treatment outcome was assessed as ineffective in $71.16 \%$ patients in the control group. The total effective rates in the treatment and control groups were 81.67 and $28.84 \%$, respectively $(\mathrm{P}<0.05$; Table III). At 4-month follow up, $60.06 \%$ patients in the treatment group exhibited marked improvement compared with $8.09 \%$ in the control group. Treatment outcome in $59.19 \%$ of patients in the control group was rated as ineffective. The total effective rates in the treatment and control groups were 85.11 and $40.81 \%$, respectively $(\mathrm{P}<0.05$; Table III). Similarly, at 6-month follow up, lesions in the majority of patients $(68.74 \%)$ in the treatment group exhibited marked improvement (Fig. 3C), whereas the outcome of ornidazole treatment alone was rated as effective in $2.87 \%$ and ineffective in $44.18 \%$ patients (Fig. 4C). At 6 months, although 83 patients were lost to follow-up, the total effective rates in the treatment and control groups were 96.56 and $55.82 \%$, respectively $(\mathrm{P}<0.05$; Table III). The total efficiency rates for the majority of lesion indicators between the two groups were statistically significant $(\mathrm{P}<0.05)$, with the exception of telangiectasia (Table IV), which indicated that application of rbFGF gel significantly improved the skin lesions.

At the end of the follow-up period, primary features including erythema, papules, desquamation and dry appearance 

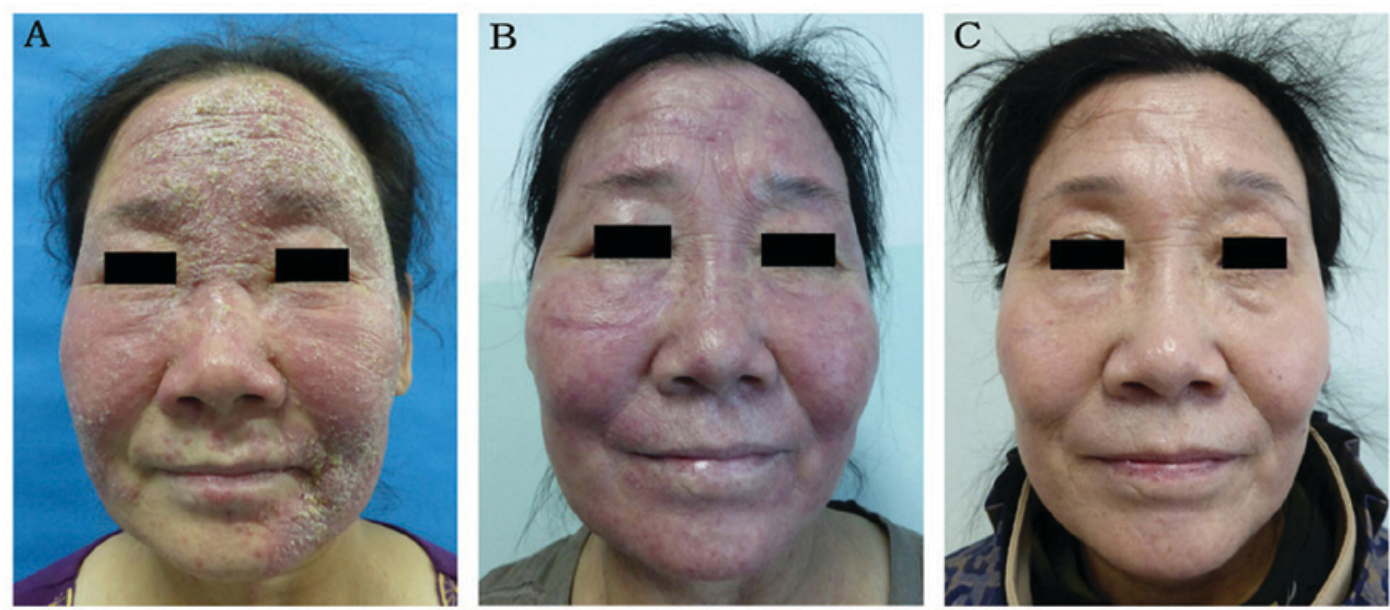

Figure 3. A female patient with rosacea in the treatment group at 8 weeks following ornidazole treatment. (A) Prior to treatment. (B) Follow up at 2 months. (C) Follow-up at 6 months.
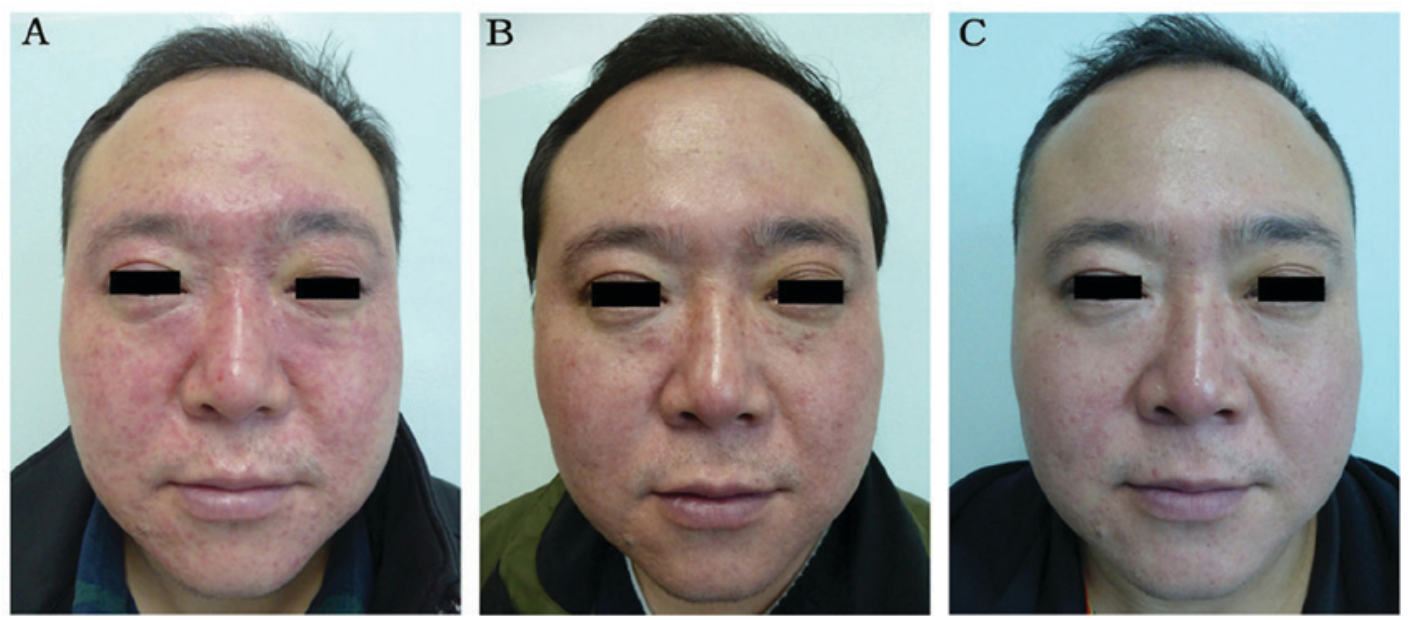

Figure 4. A male patient with rosacea in the control group at 8 weeks following ornidazole treatment. (A) Prior to treatment. (B) Follow up at 2 months. (C) Follow-up at 6 months.

prior to and following the treatment were analyzed using the Wilcoxon sign rank test $(\mathrm{P}<0.05)$. During the 6-month follow up period, none of the patients in the treatment group developed ulceration or scar formation; in contrast, $61 \%$ of patients in the control group exhibited exacerbation of skin lesions, of which $12 \%$ developed ulceration and received rbFGF treatment for a mean duration of 4 weeks. Other patients in the control group also received rbFGF treatment for $2-8$ weeks following the completion of the observation period. None of these patients experienced scar formation. This suggested that the topical use of rbFGF gel prevented scar formation.

Histological examination of facial lesions was suggestive of the presence of Demodex mite in the dermis (Fig. 5A). Key findings were epidermal hyperplasia, lymphocyte infiltration in the dermis, epithelioid cells and multinucleated giant cell granulomas (Fig. 5B). Treatment with rbFGF treatment markedly alleviated epidermal hyperplasia and reduced the size of granuloma at 2 weeks (Fig. 5C). The epidermis returned to normal at week 6 , the dermal granulomas were resolved and a large number of telangiectatic lesions became dilated (Fig. 5D).

\section{Discussion}

High infection rates of Demodex have recently been observed in patients with rosacea; the pathogenetic mechanisms of the disease mainly comprise mechanical stimulation, secondary bacterial infection and immune pathology (5). It has also been suggested that ornidazole-based therapy is highly effective in the treatment of mite folliculitis (6). Therefore, ornidazole is preferred over metronidazole due to its longer biological half-life and fewer side-effects (8). However, the current treatment is not satisfactory with respect to repair of rosacea-associated skin lesions (17). In the present study, the use of rbFGF gel was evaluated for the promotion of healing and prevention of facial scarring associated with rosacea lesions following anti-Demodex treatment.

The U.S. Food and Drug Administration (FDA) has approved several drugs for topical use in treating rosacea, such as metronidazole, azelaic acid, ivermectin, and brimonidine tartrate (18). The only approved oral drug for rosacea is low-dose doxycycline (18-21). Local treatment is typically prescribed initially, as topical use of metronidazole, sebacic 
acid and ivermectin can improve pimples and pustular rosacea (21). Metronidazole is a synthetic anti-microbial substance used for the treatment of infections caused by anaerobic bacteria and protozoa; azelaic acid is an endogenous dicarboxylic acid with anti-inflammatory properties, the effect of which is comparable to or superior than metronidazole (22). The effect of topical ivermectin was slightly better than that of topical metronidazole in the treatment of papular pustular rose acne, but both led to relapse in $\sim 66 \%$ of patients within 36 weeks following discontinuation of therapy (23). The brimonidine gel is a selective $\alpha 2$-adrenergic receptor agonist with vasoconstrictive activity for topical treatment of persistent facial erythema associated with rosacea. However, caution is advised in patients with depression, cardiovascular disease, Raynaud's phenomenon and orthostatic hypotension (24).

In our recent study intramuscular injection of compound betamethasone injection was used for patients with aggravated rosacea skin lesions, and it was able to obtain better therapeutic effects (8). However, attention should be paid to the side effects of systematically using glucocorticoids, such as local skin pigmentation, delayed healing of skin ulcers, sodium and water retention, hypokalemia and osteoporosis; whereas glucocorticoids should not be used when contraindications exists, such as infection, hypertension, diabetes, glaucoma and other diseases. RbFGF gel is a better choice for patients with rosacea and complications with these diseases, as it avoids the side-effects of glucocorticoids.

FGFs have been demonstrated to serve an important role in the repair of damaged skin. Wound repair in fibroblast growth factor receptor 1 and 2 knockout mice was delayed (25). bFGF (also referred to as FGF2) is a mitogenic cationic polypeptide protein containing 155 amino acids. Its functional domains consist of a receptor binding domain and a heparin-binding domain. bFGF binds to the receptor on the target cell, contributes to the formation, development and maturation of the skin and its appendages, and serves an important role in skin regeneration and wound repair $(26,27)$. Consistent with these findings, the present data also demonstrated that application of rbFGF alleviated erythema, papules, desquamation and dryness; it is especially useful in the treatment of rosacea granuloma lesions and prevents late scar formation. This may be explained by the following mechanisms: ii) Chemotaxis of inflammatory cells and fibroblasts into the wound; ii) promotion of neovascularization at the wound site; iii) regulation of extracellular matrix formation and degradation; and iv) stimulation of adjacent cells to produce cytokines. In addition, rbFGF has been demonstrated to promote proliferation, repair and regeneration of cells of ectodermal, neuroectodermal and mesodermal origin (such as epithelial cells, vascular endothelial cells, dermal cells and fibroblasts), promote capillary regeneration, improve local circulation and promote wound healing $(8,9,28)$. It also helps repair skin barrier structure in patients with hormone-dependent dermatitis, reduces skin sensitivity, improves skin tolerance, increases capillary elasticity and reduces capillary permeability.

In the pathological analysis of the patient samples, neutrophil activity was not observed. In addition, there was no purulent discharge in the facial lesions.

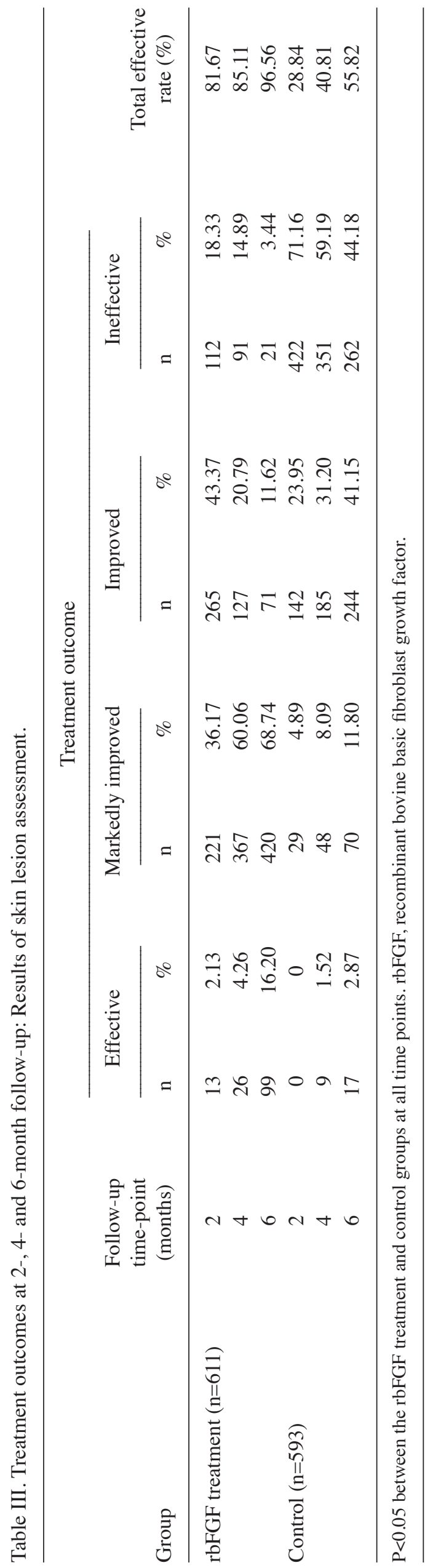


Table IV. Comparison of total effective rate using lesion indicators between the treatment and control groups at 6-month follow up.

\begin{tabular}{|c|c|c|c|c|c|c|c|c|c|c|}
\hline \multirow[b]{2}{*}{ Group } & \multicolumn{2}{|c|}{ Erythema } & \multicolumn{2}{|c|}{ Papules } & \multicolumn{2}{|c|}{ Desquamation } & \multicolumn{2}{|c|}{ Dryness } & \multicolumn{2}{|c|}{ Telangiectasia } \\
\hline & $\mathrm{n} /$ total & $\%$ & $\mathrm{n} /$ total & $\%$ & $\mathrm{n} /$ total & $\%$ & $\mathrm{n} /$ total & $\%$ & $\mathrm{n} /$ total & $\%$ \\
\hline $\begin{array}{l}\text { rbFGF } \\
\text { treatment }\end{array}$ & $243 / 320$ & 76 & $93 / 291$ & 32 & $527 / 586$ & 90 & $551 / 593$ & 93 & $47 / 223$ & 21 \\
\hline Control & $70 / 317$ & 22 & $68 / 297$ & 23 & $267 / 524$ & 51 & $323 / 548$ & 59 & $57 / 251$ & 23 \\
\hline P-value & $<0.05$ & & $<0.05$ & & $<0.05$ & & $<0.05$ & & $>0.05$ & \\
\hline
\end{tabular}

Effective index is defined as [total score (pre-rbFGF treatment-post-rbFGF treatment)/pre-treatment total score] x100. rbFGF, recombinant bovine basic fibroblast growth factor.
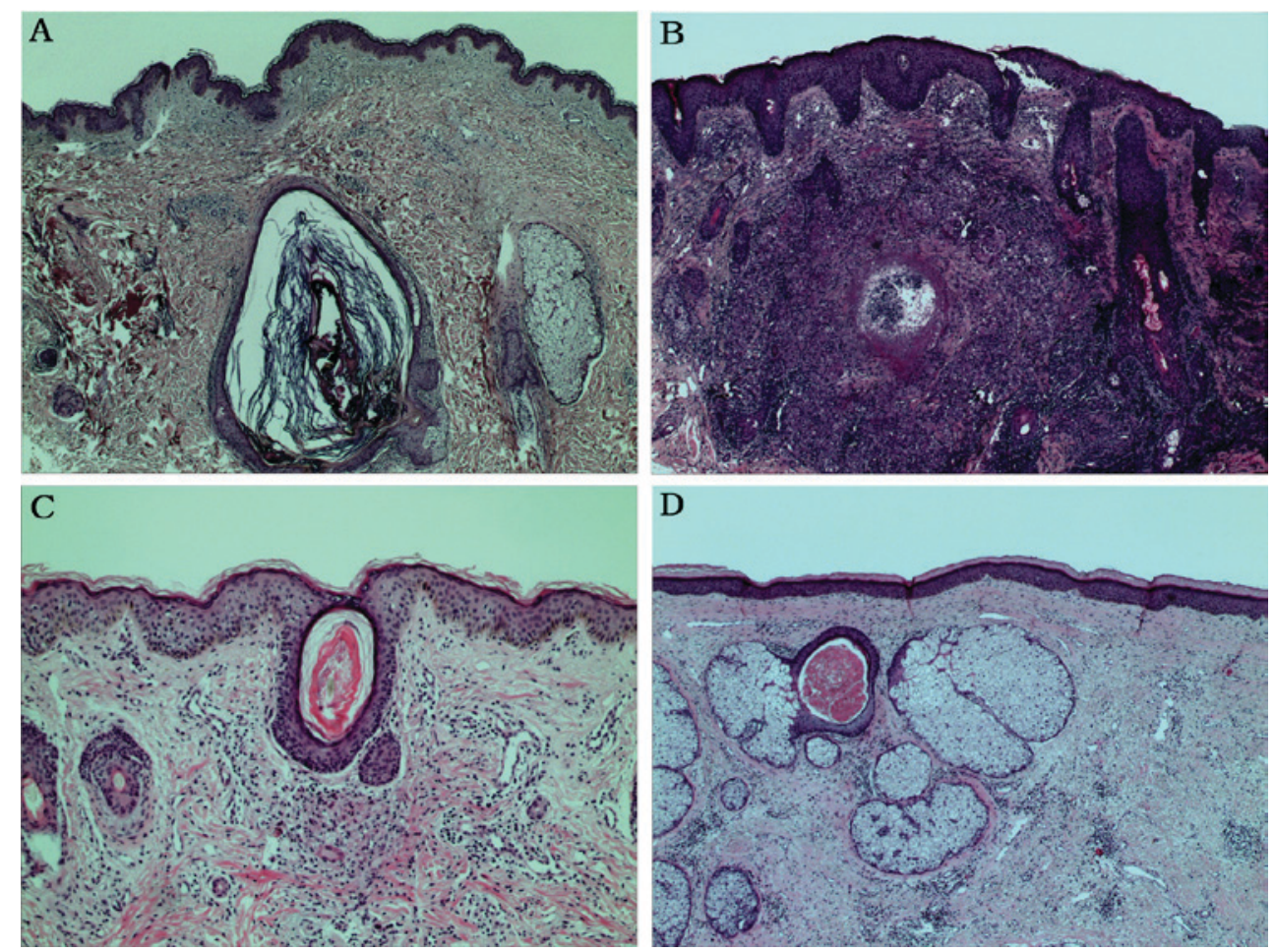

Figure 5. Hematoxylin-eosin staining of the skin biopsy. (A) Demodex mites are observed in the dermis of the rosacea patient (magnification, x40). (B) Epidermal hyperplasia and granuloma infiltration with lymphocyte, epithelioid cells and multinucleated cells in the dermis are observed (magnification, x40). (C) Treatment with rbFGF gel alleviated the hyperplasia of epidermis and reduced the size of granuloma (magnification, x100). (D) Following the rbFGF gel treatment, the epidermis has become normal, the granuloma resolved and several dilated blood vessels were observed (magnification, $\mathrm{x} 40$ ). rbFGF, recombinant bovine basic fibroblast growth factor.

Culture of necrotic tissue secretions did not produce Staphylococcus aureus and Staphylococcus epidermidis. Finally, the treatment using $2 \%$ mupirocin and $2 \%$ fusidic acid ointment all failed. Only the treatment with compound betamethasone injection (betamethasone dipropionate $5 \mathrm{mg} / \mathrm{ml}$ and betamethasone sodium phosphate $2 \mathrm{mg} / \mathrm{ml}$ ) and rbFGF gel was effective. The pathological changes following rosacea include superficial dermal tissue necrosis, caseous necrosis and formation of granuloma structure. The application of rbFGF gel was demonstrated to effectively repair the pathological structure of rosacea tissue.

The present results suggested that the effect of rbFGF gel treatment on the rosacea skin lesions following anti-Demodex were more pronounced as the treatment was extended. This could be explained by the fact that exogenous bFGF not only promotes the secretion of endogenous bFGF, but also stimulates the extracellular matrix and fibroblast protein synthesis and formation of collagen. However, there was no significant improvement in telangiectasia, which may be associated with the expansion of the blood vessels by vascular endothelial growth factor, angiogenic factor, FGF, transforming growth factor and other disorders (29).

In the present study, the effective rate was lower than that in our previous study, which may be attributable to several causes: The higher efficacy rate in the previous study may have been due to the relatively small sample size $(n=14)$, compared with the larger sample size in the present study $(n=1,204)$. Difference in the sample size may result in variable effective 
rate. Furthermore, the patients enrolled in the present study had symptoms of erythema, papules, desquamation, dryness and telangiectasia, which suggests a more severe state of disease; this may also explain the relatively lower effective rate.

Unfortunately, there was no internal control due to the disapproval of the Ethics Committee of Lanzhou General Hospital. As an alternative, an rbFGF gel vehicle control was used as and external control, in accordance with previous studies (30-32). Most importantly, the effectiveness of rbFGF treatment was evaluated by the pathological changes in the patients prior to and following the treatment.

The present single-blind study was focused on Demodex mite-induced rosacea manifested with papules and pustule. All patients included in the study were classified as moderate to severe rosacea with primary features including transient and non-transient erythema, papules and pustules and telangiectasia (12). As such, patients were not further divided into subtypes. In the patients' interest, doctors were allowed to decide whether the patients from the control group should be moved to the treatment group. The disadvantages of the single-blind study are similar to those of double-blind studies and include potential influence on data collection, analysis by investigators and the cross-over effect (33).

The cause of rosacea is unknown, but it may be due to a variety of hereditary and environmental factors $(1,18)$. Our future studies will explore the effect of rbFGF on the Demodex-mite negative rosacea induced skin lesions and its underlying mechanisms. The sample size may also be increased for pathological analysis. A further double-blind study with longer duration of follow-up and independent evaluators will be conducted in the near future to validate the present findings.

In conclusion, rbFGF may repair skin ulceration and scar formation in patients with rosacea skin lesions. Early introduction and long duration of recombinant bovine basic fibroblast growth factor gel may accelerate the effective treatment of papules, desquamation and dryness.

\section{Acknowledgements}

Not applicable.

\section{Funding}

No funding was received.

\section{Availability of data and materials}

The datasets used and/or analyzed during the current study are available from the corresponding author on reasonable request.

\section{Authors' contributions}

YL designed the experiments and performed pathological experiments. XLL collected and interpreted the data. YJS collected the follow-up data. LZ analyzed the data. JHZ collected and analyzed the data, and wrote the manuscript. These authors contributed equally to this work.

\section{Ethics approval and consent to participate}

The present study was approved by the Ethics Committee of Lanzhou General Hospital (Lanzhou, China) and all patients provided written informed consent.

\section{Patient consent for publication}

All patients provided written informed consent.

\section{Competing interests}

The authors declare that they have no competing interests.

\section{References}

1. Weinkle AP, Doktor V and Emer J: Update on the management of rosacea. Clin Cosmet Investig Dermatol 8: 159-177, 2015.

2. Gallo RL, Granstein RD, Kang S, Mannis M, Steinhoff M, Tan J and Thiboutot D: Standard classification and pathophysiology of rosacea: The 2017 update by the National Rosacea Society Expert Committee. J Am Acad Dermatol 78: 148-155, 2018.

3. Egeberg A, Hansen PR, Gislason GH and Thyssen JP: Patients with Rosacea have increased risk of depression and anxiety disorders: A Danish Nationwide Cohort Study. Dermatology 232: 208-213, 2016.

4. Libon F, El Hayderi L, Nikkels-Tassoudji N, Dezfoulian B and Nikkels AF: Rosacea. Rev Med Liege 70: 179-185, 2015 (In French).

5. Gonzalez-Hinojosa D, Jaime-Villalonga A, Aguilar-Montes G and Lammoglia-Ordiales L: Demodex and rosacea: Is there a relationship? Indian J Ophthalmol 66: 36-38, 2018.

6. Cohen PR: Follicular contact dermatitis revisited: A review emphasizing neomycin-associated follicular contact dermatitis. World J Clin Cases 2: 815-821, 2014.

7. Chang YS and Huang YC: Role of Demodex mite infestation in rosacea: A systematic review and meta-analysis. J Am Acad Dermatol 77: 441-447.e6, 2017.

8. Luo Y, Sun YJ, Zhang L and Luan XL: Treatment of mites folliculitis with an ornidazole-based sequential therapy: A randomized trial. Medicine (Baltimore) 95: e4173, 2016.

9. Zarei $\mathrm{F}$ and Soleimaninejad $\mathrm{M}$ : Role of growth factors and biomaterials in wound healing. Artif Cells Nanomed Biotechnol: $1-6,2018$

10. Jinfeng L, Yunliang W, Xinshan L, Shanshan W, Chunyang X, Peng X, Xiaopeng Y,Zhixiu X, Honglei Y, Xia C, et al: The effect of MSCs derived from the human umbilical cord transduced by fibroblast growth factor-20 on Parkinson's disease. Stem Cells Int 2016: 5016768, 2016.

11. Meduri A, Scorolli L, Scalinci SZ, Grenga PL, Lupo S, Rechichi $M$ and Meduri E: Effect of the combination of basic fibroblast growth factor and cysteine on corneal epithelial healing after photorefractive keratectomy in patients affected by myopia. Indian J Ophthalmol 62: 424-428, 2014.

12. Wilkin J, Dahl M, Detmar M, Drake L, Feinstein A, Odom R and Powell F: Standard classification of rosacea: Report of the National Rosacea Society Expert Committee on the classification and staging of rosacea. J Am Acad Dermatol 46: 584-587, 2002.

13. Rong L, Ding T and Zhou Y: Different methods in the treatment of rosacea and effect observation of staging. Sichuan Medical J 35: 444-445, 2014.

14. Tan JK: Evaluation of clinical severity by acne grading and lesion counting: Springer Berlin, Heidelberg, pp. 325-330, 2014.

15. Luo Y, Zhang LI, Sun YJ, Du H and Yang GL: Syringotropic mycosis fungoides responding well to VELP chemotherapy: A case report. Exp Ther Med 11: 2254-2258, 2016.

16. Alebachew T, Yismaw G, Derabe A and Sisay Z: Staphylococcus aureusburn wound infection among patients attending yekatit 12 hospital burn unit, addis ababa, ethiopia. Ethiop J Health Sci 22: 209, 2012.

17. Del Rosso JQ, Tanghetti EA, Baldwin HE, Rodriguez DA and Ferrusi IL: The burden of illness of erythematotelangiectatic rosacea and papulopustular rosacea: Findings from a web-based survey. J Clin Aesthet Dermatol 10: 17-31, 2017. 
18. Rainer BM, Kang S and Chien AL: Rosacea: Epidemiology, pathogenesis, and treatment. Dermatoendocrinol 9: e1361574, 2017.

19. Mikkelsen CS, Holmgren HR, Kjellman P, Heidenheim M, Kappinnen A, Bjerring P and Huldt-Nystrøm T: Rosacea: A clinical review. Dermatol Reports 8: 6387, 2016.

20. Tüzün Y, Wolf R, Kutlubay Z, Karakuş O and Engin B: Rosacea and rhinophyma. Clin Dermatol 32: 35-46, 2014.

21. Oge LK, Muncie HL and Phillips-Savoy AR: Rosacea: Diagnosis and treatment. Am Fam Physician 92: 187-196, 2015.

22. van Zuuren EJ, Fedorowicz Z, Carter B, van der Linden MM and Charland L: Interventions for rosacea. Cochrane Database Syst Rev: CD003262, 2015.

23. Ebbelaar CCF, Venema AW and Van Dijk MR: Topical ivermectin in the treatment of papulopustular rosacea: A systematic review of evidence and clinical guideline recommendations. Dermatol Ther (Heidelb) 8: 379-387, 2018.

24. Piwnica D, Pathak A, Schäfer G and Docherty JR: In vitro safety pharmacology profiling of topical $\alpha$-adrenergic agonist treatments for erythema of rosacea. Drugs R D 18: 87-90, 2018.

25. Piwnica D, M Pathak A, Schafer G and Docherty JR: In vitro safety pharmacology profiling of topical alpha-adrenergic agonist treatments for erythema in wounded skin. J Cell Sci 125: 5690-5701, 2012.

26. Zbinden A, Browne S, Altiok EI, Svedlund FL, Jackson WM and Healy KE: Multivalent conjugates of basic fibroblast growth factor enhance in vitro proliferation and migration of endothelial cells. Biomater Sci 6: 1076-1083, 2018.

27. Chen G, Ren J, Deng Y, Wu X, Huang J, Wang G, Zhao Y and Li J: An injectable, wound-adapting, self-healing hydrogel for fibroblast growth factor 2 delivery system in tissue repair applications. J Biomed Nanotechnol 13: 1660-1672, 2017.
28. Qu Y, Cao C, Wu Q, Huang A, Song Y, Li H, Zuo Y, Chu C, Li J and Man Y: The dual delivery of KGF and bFGF by collagen membrane to promote skin wound healing. J Tissue Eng Regen Med 12: 1508-1518, 2018.

29. Marcelo KL, Goldie LC and Hirschi KK: Regulation of endothelial cell differentiation and specification. Circ Res 112: 1272-1287, 2013

30. Taieb A, Ortonne JP, Ruzicka T, Roszkiewicz J, Berth-Jones J, Peirone $\mathrm{MH}$ and Jacovella J; Ivermectin Phase III study group: Superiority of ivermectin $1 \%$ cream over metronidazole $0.75 \%$ cream in treating inflammatory lesions of rosacea: A randomized, investigator-blinded trial. Br J Dermatol 172: 1103-1110, 2015.

31. DuBois J, Dover JS, Jones TM, Weiss RA, Berk DR and Ahluwalia G: Phase 2 randomized, dose-ranging study of oxymetazoline cream for treatment of persistent facial erythema associated with rosacea. J Drugs Dermatol 17: 308-316, 2018.

32. Gold LS, Papp K, Lynde C, Lain E, Gooderham M, Johnson S and Kerrouche N: Treatment of rosacea with concomitant use of topical ivermectin $1 \%$ cream and brimonidine $0.33 \%$ gel: A randomized, vehicle-controlled study. J Drugs Dermatol 16: 909-916, 2017.

33. Friedman LM, Furberg CD, DeMets D, Reboussin DM and Granger CB: Fundamentals of clinical trials. Springer, 2015. International (CC BY-NC-ND 4.0) License. 